\title{
Getting Covariantly Smeared Sources into Better Shape
}

\author{
Georg von Hippel*, Benjamin Jäger, Thomas Rae and Hartmut Wittig \\ PRISMA Cluster of Excellence and Institut für Kernphysik, University of Mainz, 55099 Mainz, \\ Germany \\ E-mail: hippel@kph.uni-mainz.de
}

\begin{abstract}
The use of covariantly smeared sources in hadronic correlators is a common method of improving the projection onto the ground state. Studying the dependence of the shape of such sources on the gauge field background, we find that localized fluxes of magnetic field can strongly distort the sources. This results in a reduction of the smearing radii that can be reached by iterative smearing prescriptions, in particular as the continuum limit is approached. As a remedy, we propose a novel covariant smearing procedure ("free-form smearing") enabling the creation of arbitrarily shaped sources, including in particular Gaussians of arbitrary radius, as well as shapes with nodes, such as hydrogenic wavefunctions without the need to fix a gauge.
\end{abstract}

31st International Symposium on Lattice Field Theory LATTICE 2013

July 29 - August 3, 2013

Mainz, Germany

\footnotetext{
*Speaker.
} 


\section{Introduction}

In measuring hadronic correlation functions, the use of interpolating operators with good projection properties onto the ground state is crucial in order to minimize unwanted contributions from excited states. A widely-used technique is the use of covariantly smeared quark fields as part of the construction of interpolating operators. Covariant smearing is typically implemented in an iterative fashion by defining a smeared field $[1,2,3]$

$$
\widetilde{\psi}=C(1+\alpha H)^{n} \psi
$$

wich approximates a Gaussian shape in the free-field case.

This leads to the questions of what shapes this smearing prescription produces when used on actual gauge ensembles, where strong interactions are present; and how to deal with cases where a different shape is desirable. In a recent paper [4], we have given answers to these questions.

\section{Covariant smearing}

A generic covariant smearing operation can be written as

$$
\widetilde{\psi}(x)=\sum_{y} K(x, y) \psi(y)
$$

with a kernel $K$ that can be decomposed into paths via

$$
K(x, y)=\sum_{\mathscr{P} \in P(x, y)} \omega_{\mathscr{P}} U_{\mathscr{P}}
$$

where $P(x, y)$ is some set of paths from $x$ to $y$ and

$$
U_{\mathscr{P}} \equiv U_{\mu_{\mathscr{P}, 1}}(x) \cdots U_{\mu_{\mathscr{P}, l \mathscr{P}}}\left(x+\sum_{j=1}^{l_{\mathscr{P}}-1} \hat{e}_{\mu_{\mathscr{P}, j}}\right)
$$

is the product of links along $\mathscr{P}$.

For the most usual case of a point source $\psi(y)=\phi_{0} \delta\left(y-y_{0}\right)$, this reduces to

$$
\widetilde{\psi}(x)=\sum_{\mathscr{P} \in P\left(x, y_{0}\right)} \omega_{\mathscr{P}} U_{\mathscr{P}} \phi_{0} .
$$

The norm of the source, which enters the smearing radius via

$$
r_{\mathrm{sm}}^{2}=\frac{\sum_{x}\left|x-y_{0}\right|^{2}\|\tilde{\psi}(x)\|^{2}}{\sum_{x}\|\tilde{\psi}(x)\|^{2}}
$$

is then given by

$$
\begin{aligned}
\|\widetilde{\psi}(x)\|^{2} & =\left\|\sum_{\mathscr{P} \in P\left(x, y_{0}\right)} \omega_{\mathscr{P}} U_{\mathscr{P}} \phi_{0}\right\|^{2} \\
& =\sum_{\mathscr{P} \in P\left(x, y_{0}\right)} \omega_{\mathscr{P}}^{2}\left\|\phi_{0}\right\|^{2}+\sum_{\mathscr{P}_{i} \neq \mathscr{P}_{j} \in P(x, 0)} \omega_{\mathscr{P}_{i}} \omega_{\mathscr{P}_{j}} \phi_{0}^{\dagger} U_{\mathscr{P}_{i}}^{\dagger} U_{\mathscr{P}_{j}} \phi_{0}
\end{aligned}
$$

and thus depends on the magnetic flux through the surfaces spanned by the paths in $P(x, y)$ [4]. 

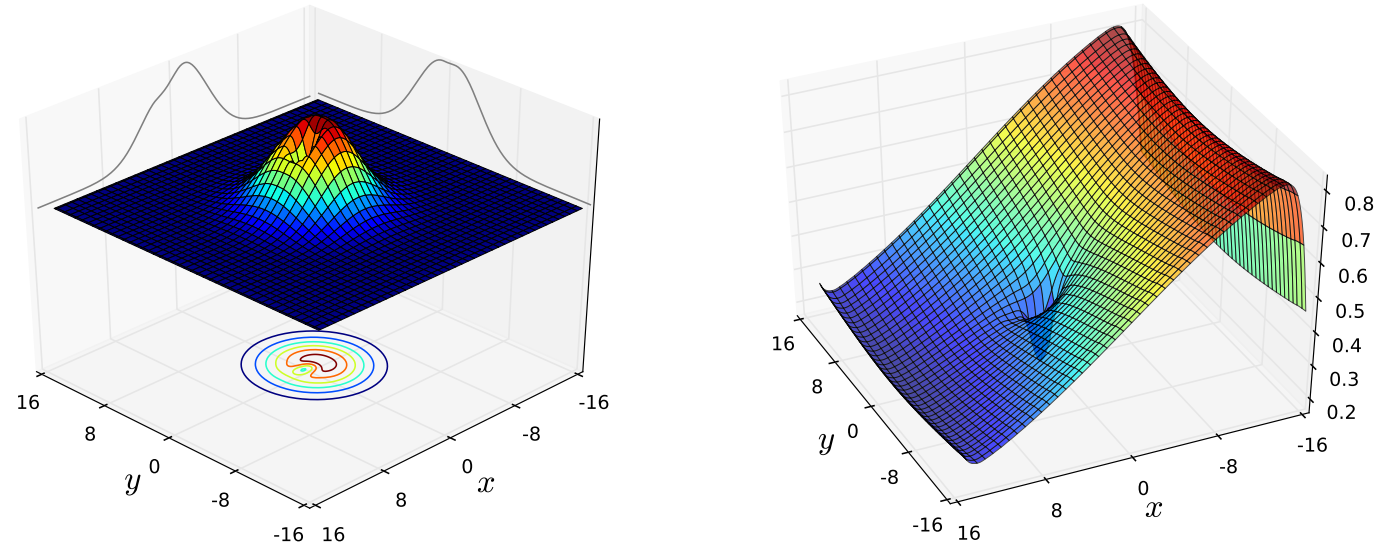

Figure 1: Distortion of a Gaussian source on a unit configuration with a single non-unit link; left: the shape of the source, right: the ratio of the source to its counterpart on a pure unit configuration.
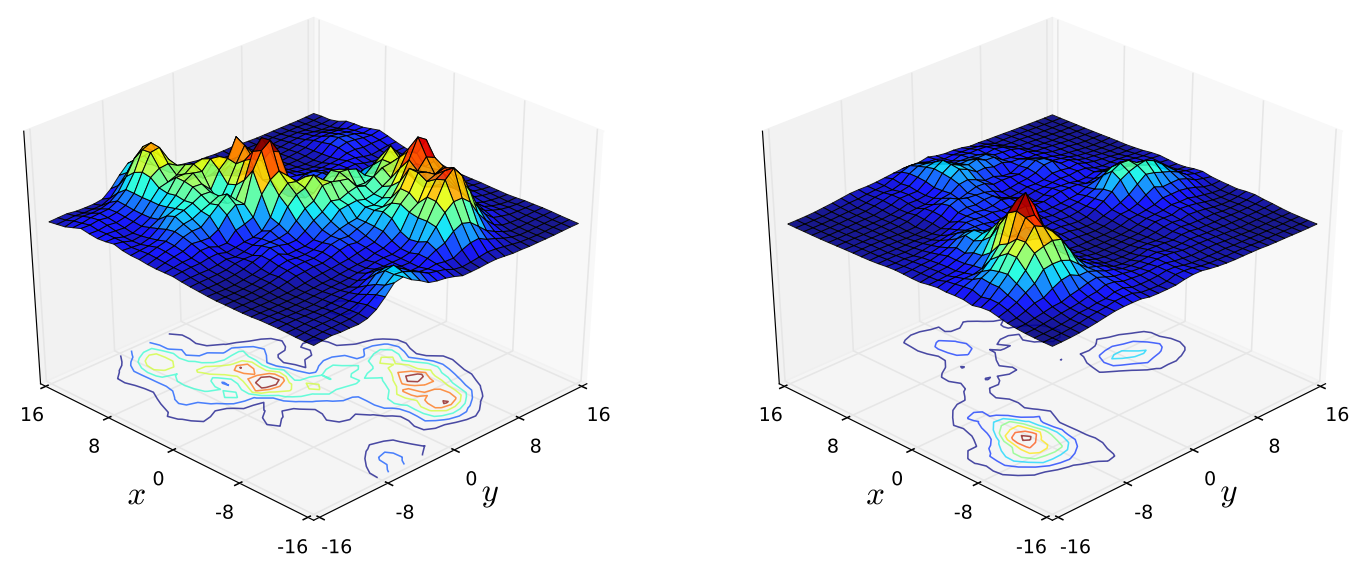

Figure 2: Examples of distorted source shapes observed on actual configurations. The canonical shape should be a wide, centered Gaussian in each case.

\section{The shape of sources}

The dependence of the source shape on the gauge field can be shown most easily in an artificial setup: in fig. 1, we show the shape of a covariantly smeared source on a gauge configuration with all but one link set to unity. It can be seen that the magnetic flux through the plaquettes containing the non-unit link leads to both a strong local suppression of the smeared source and to an overall shift of the source compared to its counterpart on a unit configuration.

As a consequence of such shifts and suppressions, iteratively smeared sources tend to take bizarre, multimodal, and strongly non-Gaussian shapes on typical configurations (cf. fig. 2), in particular as the iteration count $n$ in eq. (1.1) is increased.

Averaging over such distorted sources leads to an observed decrease of the achievable smearing radii with decreasing lattice spacing, as can be seen in the "smearscapes" (plots of $r_{\mathrm{sm}}$ against 

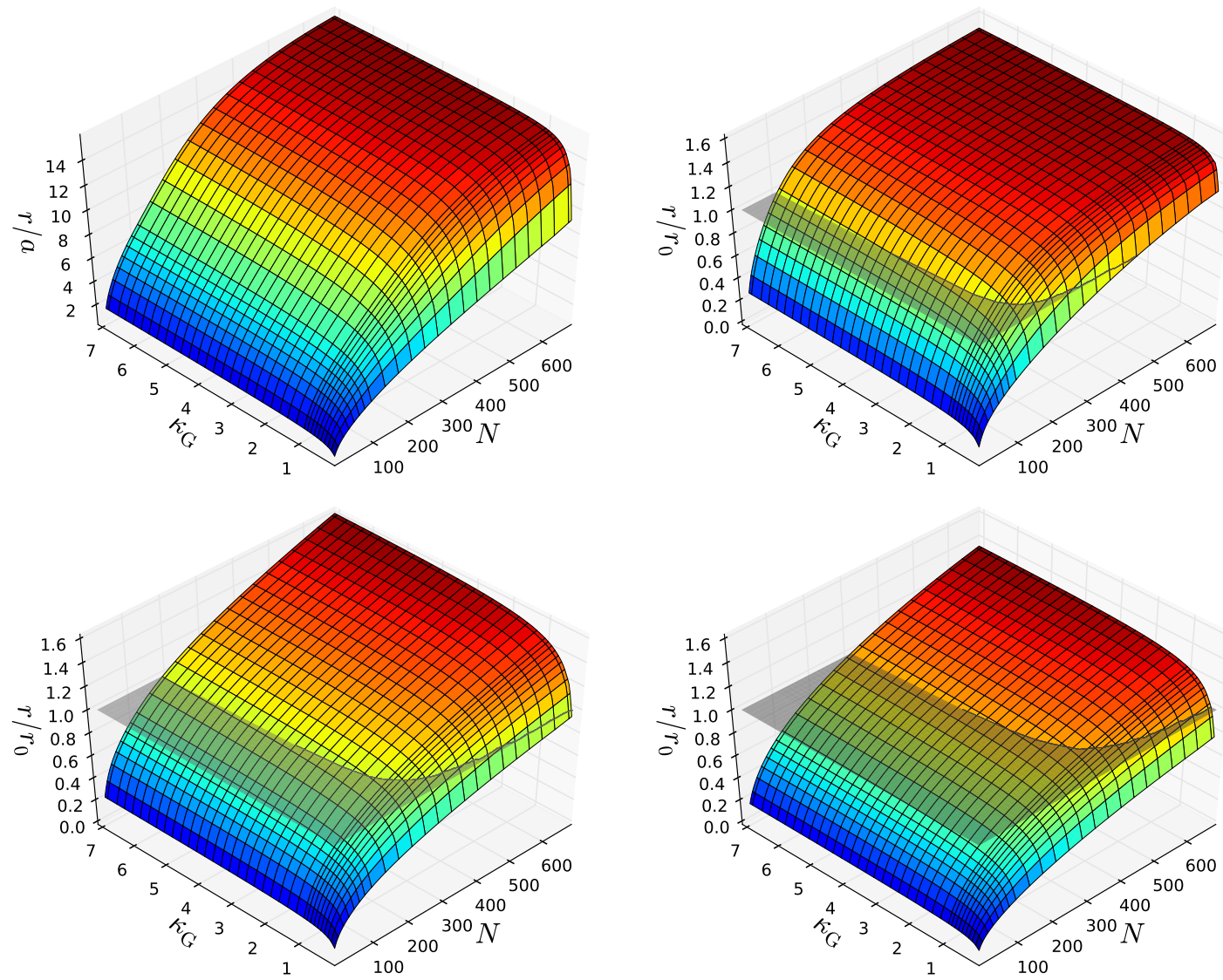

Figure 3: "Smearscapes" demonstrating the dependence of the smearing radius on the smearing parameters $\left(\kappa_{\mathrm{G}}=\frac{\alpha}{1-\alpha}, n\right)$ for the free case (top left) and for a range of lattice spacings (top right: $a \approx 0.076 \mathrm{fm}$, bottom left: $a \approx 0.066 \mathrm{fm}$, bottom right: $a \approx 0.049 \mathrm{fm}$ ). The radius corresponding to $r_{\mathrm{sm}}=0.5 \mathrm{fm}$ is shown as a semi-transparent plane.

$\kappa_{\mathrm{G}}$ and $n$ ) of fig. 3. As the lattice spacing $a$ is decreased, the iteration counts required to reach $r_{\mathrm{sm}} \approx 0.5 \mathrm{fm}$ increase rapidly, making bizarrely distorted source shapes more and more dominant.

It has been known for a while that large smearing radii are unattainable without the use of link smearing in the smearing kernel [7]. With link smearing, larger radii can be reached, but, as the comparison in fig. 4 shows, the shapes of the sources do not become less distorted for moderate amounts of smearing. (Except for the two plots shown in fig. 4, all results shown employ a single iteration of APE link smearing [5, 6]).

The strong distortion of the sources throws doubt on the usefulness of covariant smearing as an effective means of suppressing excited state contributions, since it is unclear why the projection on the ground state should be particularly good for these odd shapes.

\section{Free-form smearing}

It is therefore desirable to find a method that allows one to covariantly smear sources with large 

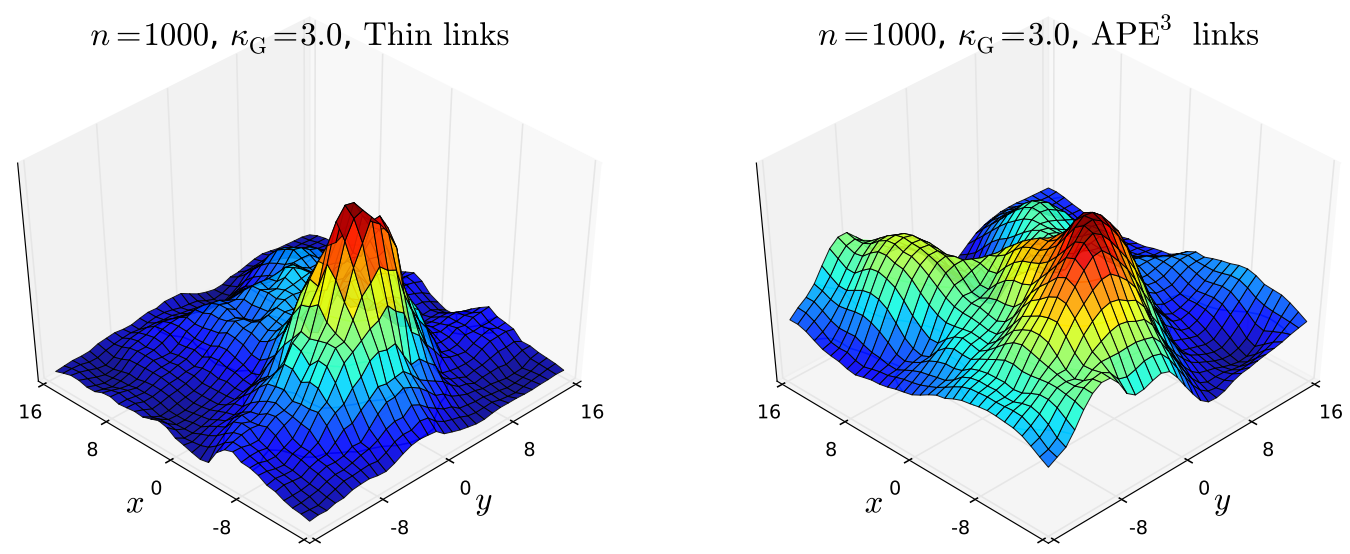

Figure 4: An example of the effect of link smearing on the shape of an iteratively smeared source: left: using thin links, right: using three iterations of APE [5, 6] link smearing on the same configuration with all other parameters exactly the same.
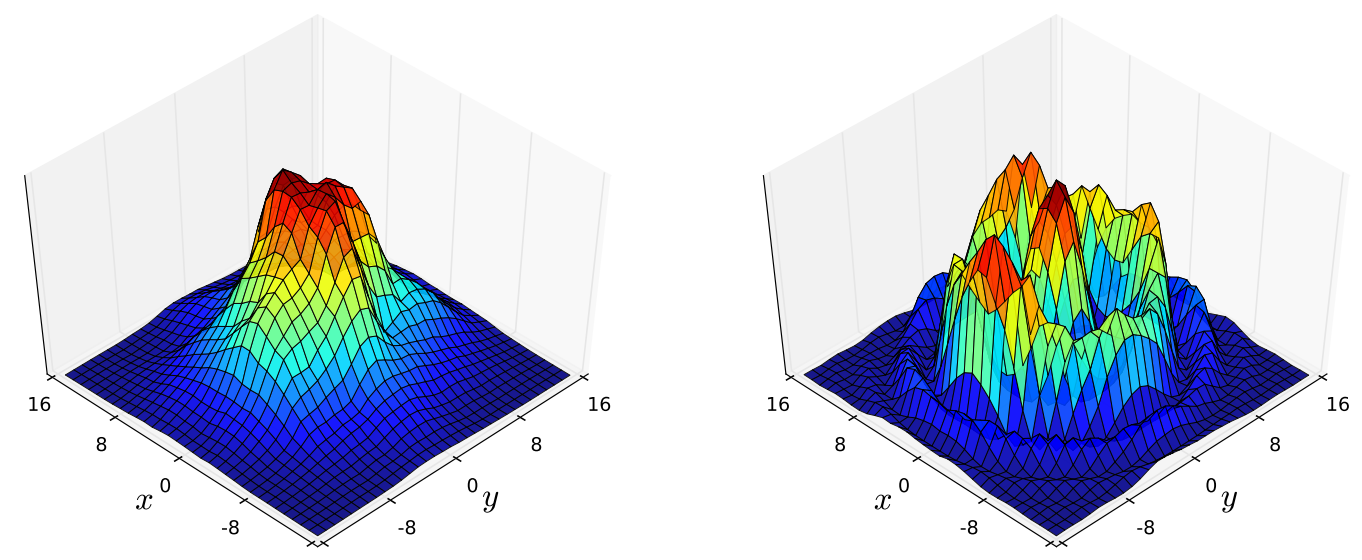

Figure 5: Examples of free-form smeared sources; left: a Gaussian shape, right: a Gaussian modulated by radial oscillations, as an example of a source shape with nodes.

smearing radii. Another feature that would be highly desirable in a covariant smearing method would be the ability to create wavefunctions of non-Gaussian forms (e.g. hydrogen-like wavefunctions), which currently is possible only in a non-covariant gauge-fixed approach.

To achieve both of these ends, we have proposed the following method ("free-form smearing" [4]):

1. using iterative covariant smearing, calculate $\psi^{\prime}(x)=\left[(1+\alpha H)^{n}\right]_{x, y} \delta\left(y-y_{0}\right) \phi_{0}$, with $n$ chosen small, but large enough to touch all points on a timeslice,

2. determine the gauge average $N(x)=\left\langle\left\|\psi^{\prime}(x)\right\|\right\rangle$, and

3. define the free-formed smeared source as $\tilde{\psi}(x)=\psi^{\prime}(x) f\left(x-y_{0}\right) / N(x)$. 



Figure 6: Effective mass plots showing the effects of free-form smearing with Gaussian shapes of varying radius; top: pion, bottom: nucleon; the iterative smearing used to build the set of paths is shown in black.

By construction, the free-form smeared source satisfies $\langle\|\tilde{\psi}(x)\|\rangle=\left|f\left(x-y_{0}\right)\right|\left\|\phi_{0}\right\|$ in the gauge average. Note that $N(x)$ only needs to be computed once on each ensemble to be able to free-form smear with arbitrarily many shapes $f$ afterwards.

In fig. 6 , we show effective mass plots for the pion and nucleon on an $a \approx 0.066 \mathrm{fm}$ CLS ensemble. Shown in black are the effective masses for the iteratively smeared source $\psi^{\prime}$ used as the basis, while the coloured points represent free-form smeared sources $\tilde{\psi}$ of Gaussian shape with different radii. It can be seen that the use of free-form smeared sources with large radii leads to a significant suppression of excited-state contamination for the nucleon (and less so in the pion case), which allows a reliable plateau fit at much earlier times. We expect that this will make free-form smearing particularly useful for baryon spectroscopy. 


\section{Discussion}

The significant distortions of the shape of iteratively smeared covariant sources in the presence of non-trivial gauge field backgrounds limits the smearing radii which can be used, particularly so at small lattice spacing. This poses a problem particularly in baryonic channels, where suppression of excited states is especially important to provide a good plateau region.

To avoid this problem, we propose a novel smearing procedure, which we call "free-form" smearing, that enables the use not only of Gaussians of arbitrary width, but also of other source shapes not available through iterative methods of covariant smearing.

While free-form smearing in its current form cannot be readily used for sink smearing, we expect it to be extremely useful for many spectroscopic applications and plan to apply it to improving the determination of the lattice spacing using the mass of the $\Omega$ previously performed in [8].

Acknowledgments: Our calculations were performed on the "Wilson" HPC Cluster at the Institute for Nuclear Physics, University of Mainz. We thank Dalibor Djukanovic and Christian Seiwerth for technical support. We are grateful for computer time allocated to project HMZ21 on the BG/Q "JUQUEEN" computer at NIC, Jülich. This work was granted access to the HPC resources of the Gauss Center for Supercomputing at Forschungzentrum Jülich, Germany, made available within the Distributed European Computing Initiative by the PRACE-2IP, receiving funding from the European Community's Seventh Framework Programme (FP7/2007-2013) under grant agreement RI-283493. This work was supported by the DFG via SFB 1044 and grant HA 4470/3-1. The 3d plots shown in this paper were generated using matplotlib [9]. We are grateful to our colleagues within the CLS initiative for sharing ensembles.

\section{References}

[1] S. Güsken, U. Löw, K. H. Mütter, R. Sommer, A. Patel, et al., Nonsinglet Axial Vector Couplings of the Baryon Octet in Lattice QCD, Phys.Lett. B227 (1989) 266.

[2] C. Alexandrou, F. Jegerlehner, S. Güsken, K. Schilling, and R. Sommer, B meson properties from lattice QCD, Phys.Lett. B256 (1991) 60-67.

[3] UKQCD Collaboration, C. Allton et al., Gauge invariant smearing and matrix correlators using Wilson fermions at Beta = 6.2, Phys.Rev. D47 (1993) 5128-5137, [hep-lat/93030 09].

[4] G. M. von Hippel, B. Jäger, T. D. Rae, and H. Wittig, The Shape of Covariantly Smeared Sources in Lattice QCD, JHEP 1309 (2013) 014, [1306.1440].

[5] APE Collaboration, M. Albanese et al., Glueball Masses and String Tension in Lattice QCD, Phys.Lett. B192 (1987) 163-169.

[6] S. Basak, I. Sato, S. Wallace, R. Edwards, D. Richards, et al., Combining quark and link smearing to improve extended baryon operators, PoS LAT2005 (2006) 076, [hep-lat / 0509179 ].

[7] S. Syritsyn, J. Bratt, M. Lin, H. Meyer, J. Negele, et al., Nucleon Electromagnetic Form Factors from Lattice QCD using 2+1 Flavor Domain Wall Fermions on Fine Lattices and Chiral Perturbation Theory, Phys.Rev. D81 (2010) 034507, [0 907 . 4194].

[8] S. Capitani, M. Della Morte, G. von Hippel, B. Knippschild, and H. Wittig, Scale setting via the $\Omega$ baryon mass, PoS LATTICE2011 (2011) 145, [1110.6365].

[9] J. D. Hunter, Matplotlib: A 2d graphics environment, Computing In Science \& Engineering 9 (2007), no. 3 90-95. 